\title{
Notes on the paper entitled 'Generalizations of the logarithmic Hardy inequality in critical Sobolev-Lorentz spaces'
}

\author{
Shuji Machihara', Tohru Ozawa² and Hidemitsu Wadade ${ }^{3 *}$
}

\section{"Correspondence:}

wadade@se.kanazawa-u.ac.jp

${ }^{3}$ Faculty of Mechanical Engineering Institute of Science and

Engineering, Kanazawa University, Kakuma, Kanazawa, Ishikawa 920-1192, Japan

Full list of author information is available at the end of the article
The purpose of this note is to clarify the novelty of the paper entitled 'Generalizations of the logarithmic Hardy inequality in critical Sobolev-Lorentz spaces' which was published in the J. Inequal. Appl. 2013:381, 2013 [1]. After this paper was published, the authors were informed of the references [2-5], and [6], the results of which partly overlap with those of [1]. In the paper [1], the authors established the Hardy inequality of the logarithmic type in the critical Sobolev-Lorentz spaces $H_{p, q}^{\frac{n}{p}}\left(\mathbb{R}^{n}\right)$; see Section 2 in [1] for the precise definition of $H_{p, q}^{\frac{n}{p}}\left(\mathbb{R}^{n}\right)$. The main theorem in [1] is stated as follows.

Theorem A [1, Theorem 1.1] Let $n \in \mathbb{N}, 1<p<\infty, 1<q \leq \infty$ and $1<\alpha, \beta<\infty$. Then the inequality

$$
\left(\int_{\left\{|x|<\frac{1}{2}\right\}} \frac{|u(x)|^{\alpha}}{|\log | x||^{\beta}} \frac{d x}{|x|^{n}}\right)^{\frac{1}{\alpha}} \leq C\|u\|_{H_{p, q}^{\frac{n}{p}}}
$$

holds for all $u \in H_{p, q}^{\frac{n}{p}}\left(\mathbb{R}^{n}\right)$ if and only if one of the following conditions (i), (ii), and (iii) is fulfilled:

$$
\left\{\begin{aligned}
\text { (i) } 1+\alpha-\beta<0 ; \\
\text { (ii) } 1+\alpha-\beta \geq 0 \quad \text { and } q<\frac{\alpha}{1+\alpha-\beta} \text {; } \\
\text { (iii) } 1+\alpha-\beta>0, \quad q=\frac{\alpha}{1+\alpha-\beta} \text { and } \alpha \geq \beta .
\end{aligned}\right.
$$

However, the inequality (1) was established under the condition (iii) in [7] and [6]. More precisely, the particular case of $\alpha=\beta=p=q$ was considered in [7], where the authors also obtained similar inequalities in the critical Besov spaces. In [6], the authors considered Bessel potential spaces with a logarithmic smoothness on Lorentz-Zygmund spaces which extend the critical Sobolev-Lorentz spaces $H_{p, q}^{\frac{n}{p}}\left(\mathbb{R}^{n}\right)$ for instance. Indeed, the authors in [6] proved logarithmic Hardy inequalities corresponding to (1) in critical Sobolev-LorentzZygmund spaces with a logarithmic smoothness in [6, Theorem 5.1]. This theorem includes the inequality (1) under the condition (iii) as special cases.

Based on the historical remarks above, the novelty in the paper [1] is to have given other conditions (i) and (ii) with which the inequality (1) holds and to have shown that the conditions (i), (ii), and (iii) are also necessary for (1). Especially, the marginal case $q=\infty$ was 
also considered in [1], where the norm $\|u\|_{H_{p, \infty}^{\frac{n}{p}}}$ becomes smallest in the sense of SobolevLorentz spaces, and Theorem A shows that the condition (i) is necessary and sufficient for (1) to hold.

We also add other references [2-4], and [5] in this note, which are closely related to the results in [1] and which were not cited in [1].

\section{Competing interests}

The authors declare that they have no competing interests.

\section{Authors' contributions}

All authors contributed equally to the writing of this paper. All authors read and approved the final manuscript.

\section{Author details}

${ }^{1}$ Department of Mathematics, Saitama University, 255 Shimookubo, Sakuraku, Saitama, 338-8570, Japan. ${ }^{2}$ Department of Applied Physics, Waseda University, Shinjuku, Tokyo, 169-8555, Japan. ${ }^{3}$ Faculty of Mechanical Engineering, Institute of

Science and Engineering, Kanazawa University, Kakuma, Kanazawa, Ishikawa 920-1192, Japan.

Received: 22 February 2014 Accepted: 17 June 2014 Published: 22 Jul 2014

\section{References}

1. Machihara, S, Ozawa, T, Wadade, H: Generalizations of the logarithmic Hardy inequality in critical Sobolev-Lorentz spaces. J. Inequal. Appl. 2013, 381 (2013)

2. Gogatishvili, A, Neves, JS, Opic, B: Optimality of embeddings of Bessel-potential-type spaces into Lorentz-Karamata spaces. Proc. R. Soc. Edinb., Sect. A, Math. 134, 1127-1147 (2004)

3. Gogatishvili, A, Neves, JS, Opic, B: Optimality of embeddings of Bessel-potential-type spaces. In: Drábek, P, Rákosník, J (eds.) FSDONA 2004 Proceedings, pp. 97-112. Math. Inst. Acad. Sci. Czech Republic, Prague (2005)

4. Neves, JS: Lorentz-Karamata spaces, Bessel and Riesz potentials and embeddings. Diss. Math. 405, 1-46 (2002)

5. Opic, B, Trebels, W: Bessel potentials with logarithmic components and Sobolev-type embeddings. Anal. Math. 26, 299-319 (2000)

6. Opic, B, Trebels, W: Sharp embeddings of Bessel potential spaces with logarithmic smoothness. Math. Proc. Camb. Philos. Soc. 134, 347-384 (2003)

7. Edmunds, DE, Triebel, H: Sharp Sobolev embeddings and related Hardy inequalities: the critical case. Math. Nachr. 207, 79-92 (1999)

10.1186/1029-242X-2014-253

Cite this article as: Machihara et al.: Notes on the paper entitled 'Generalizations of the logarithmic Hardy inequality

in critical Sobolev-Lorentz spaces'. Journal of Inequalities and Applications 2014, 2014:253

\section{Submit your manuscript to a SpringerOpen ${ }^{\odot}$ journal and benefit from:}

- Convenient online submission

- Rigorous peer review

- Immediate publication on acceptance

- Open access: articles freely available online

- High visibility within the field

- Retaining the copyright to your article 